\title{
Analysis of Ovarian Diseases Using Ultrasound Images
}

\author{
${ }^{1}$ K.Sheikdavood, ${ }^{2}$ S.Palanivel Rajan \\ ${ }^{1}$ Assistant Professor, Department of Electronics and Communication Engineering, \\ M.Kumarasamy College of Engineering, Karur, Tamilnadu \\ Email ID : sheikdavoodece@gmail.com \\ ${ }^{2}$ Assistant Professor, Department of Electronics and Communication Engineering, \\ M.Kumarasamy College of Engineering, Karur, Tamilnadu \\ Email ID : palanivelrajanme@gmail.com
}

\begin{abstract}
Ovarian disease is the most common disease occurring in female reproductive organs. The premature recognition and action needed for diagnosis the disease to avoid infertility or cancer to them. The reason behind infertility is Polycystic Ovary Syndrome (PCOS) and the Ovarian Regenerative Tissues Cells. PCOS is the kind of endocrine disorder present in female's ovary and creates problem with Irregular menstrual periods, skin diseases, excess hair growth in body and face, acne and finally infertility. For ovarian regenerative tissues cells histopathology slides can be well stored put away in digitized color image design. One of the well known determination inclinations to recognize ovarian tissues is ultrasound image scanner. In any case, because of various shape, size and color, distinguishing proof of ovarian tissues is a testing assignment for ultrasound scanners as it procedure dark scale images. In order to overcome these diseases, we proposed techniques for ultrasound images. By this techniques follicle in the ovary and the ovarian regenerative tissues cells is automatically detected.
\end{abstract}

\section{INDEX TERMS}

Polycystic Ovarian syndrome, Ultrasound Image, Multiscale Morphological Approach, histopathology slides

\section{INTRODUCTION}

Polycystic Ovarian Syndrome, or usually known as PCOS, is one of the most recognized, unpredictable, heterogeneous endocrine issue among females of reproductive age. The precise cause behind event of PCOS is still obscure yet elements, for example, hormonal irregularity, having body-mass-index (BMI) greater than 24, central obesity or overall obesity are a few variables adding to it [14]. The foremost components are anovulation, amenorrhea and ovulation-related infertility; excessive amounts or impacts of androgenic hormones, which brings about skin break out and hirsuitism; and insulin resistance, which is frequently related obesity, with type 2 diabetes and high cholesterol level[12].There are essentially three criteria for finding of PCOS.These are clinical, biochemical, and gonad ultrasound imaging.As per it, a patient might be analyzed as suffering from PCOS if any two of the following three conditions are seen: (i) Chronic Anovulation or Oligo-ovulation described by unpredictable menstrual cycle, (ii) Excess androgen movement portrayed by event of skin break out, hirsuitism, and elevated serum compounds and (iii) Presence of polycystic ovaries seen by gynecologic ultrasound .If there should be an occurrence of typical ovary, under impact of right levels of hormones FSH and LH (i.e. Follicle Stimulating Hormone and Luteinizing Hormone),only one follicle develops in size to around $20 \mathrm{~mm}$ in breadth, develops and gets to be prepared for ovulation.PCOS influenced ovary, because of reduced levels of FSH and $\mathrm{LH}$ and large amounts of prolactine[11], follicles neglect to develop and achieve development[13,15,16]. Consequently, in ultrasound image of PCOS influenced ovary, substantial number of little follicles (ordinarily 12 or progressively and around 2-9 $\mathrm{mm}$ in width) can be seen appropriated along the periphery of the ovary, traditionally depicted as 'necklace formation. The ovarian tissue which is made by follicle can be detected by digital scanners and image processing techniques. Digital histopathology image examination has become popular technique use by pathology specialists [17]. Image investigation diminishes time, exertion, human mistakes and subjectivity for the analysis of different tissues by pathologists.

Most existing research works have concentrated on the investigation of cancerouscells.Most existing exploration work related to ovarian reproductive tissue recognition depends on the biopsy slides obtained from ovine and rats and has specified that the attributes of human ovarian tissues are not quite the same as creature ovarian tissues. An exception to this, is the work of where three human ovaries were gathered from a post-surgery includingmalignancy patients [5]. Human reproductive potential depends upon the availability of the follicles, which regularly differs inferable from hereditary qualities and different issues such medications, radiation and poisonous environmental conditions. To analyze the ovarian tissues, screening tests utilizing an electronic gadget or biopsy test example including histopathology digital images should be directed. The ultrasound screening test is considered to be a more efficient one.

\subsection{Baseline Evaluation}

A methodical way to deal with ultrasound of the pelvis starts with a complete range through the pelvis from one side to the opposite side, envisioning the cervix, uterus, ovaries, to guarantee that no part of the exam has been excluded [10]. The exam starts amid the arrangement of the endovaginal test in the vagina, and the bladder is at first assessed. The pattern assessment of the uterine body generally incorporates a few components, containing the general size in standard measurements of the uterus (length, tallness, width), position, the consistency of the endometrium, and in addition any deformities of the uterus or other pathology[19]. The uterus is regularly measured in the midsagittal plane for the 
longitudinal length and the tallness, measured in the anterior-posterior (AP) distance across (Fig. 1). The length stretches out from the end of the cervix to the highest point of the fundus.

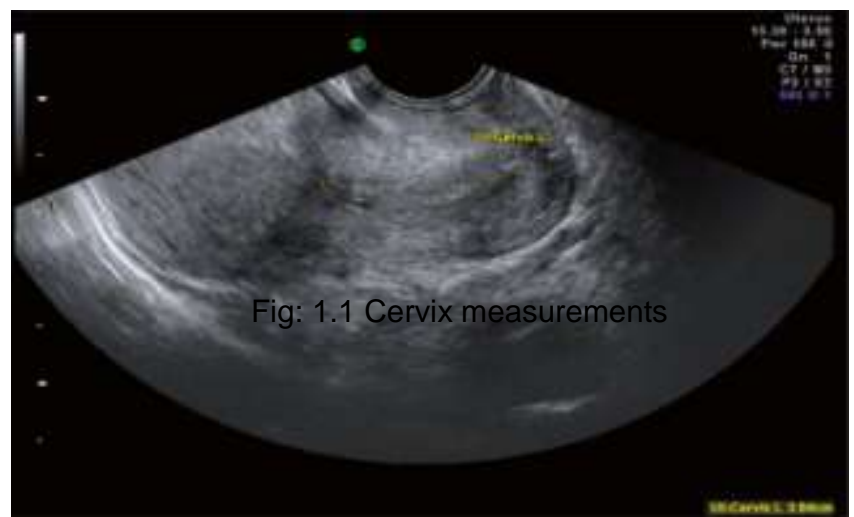

Fig 1 : Measurement of the anterior-posterior (AP) distance

The span of the uterus relates well to the general estrogenation of the body, with lower and ordinary/hoisted estrogen values corresponding with littler and bigger uterine size, respectively [3]. The presumption that littler uteri might be connected with unfavorable pregnancy results is mistaken; indeed, bigger uteri were all the more profoundly connected with ectopic pregnancies in IVF/ICSI cycles. The position of the uterus is likewise routinely recorded as a dynamic estimation [7]. This data is critical for techniques, for example, fetus exchange, which is thusly talked about; moreover, if the uterus is noted to stay unmoving on serial exams, the worry is raised for cement illness or an entangled uterus.

\section{POLYCYSTIC OVARY SYNDROME}

\subsection{Preprocessing of Ovarian Ultrasound Image}

The primary drawback of medical ultrasonography is low quality of images having low contrast and high noise content [2]. This makes automated ultrasound image processing a complicated task. In this way it is important to enhance complexity of the image and remove noise before further handling, for example, feature extraction, segmentation and follicle detection. Preprocessing of the information image includes enhancing nature of the image utilizing contrast improvement and separating procedures. Histogram Equalization procedure is utilized. As it is a worldwide differentiation upgrade method, it increases contrast of noise also along with preferred features [18]. To eliminate the additive and speckle noise present in the images different filters are used.

\subsection{Proposed method}

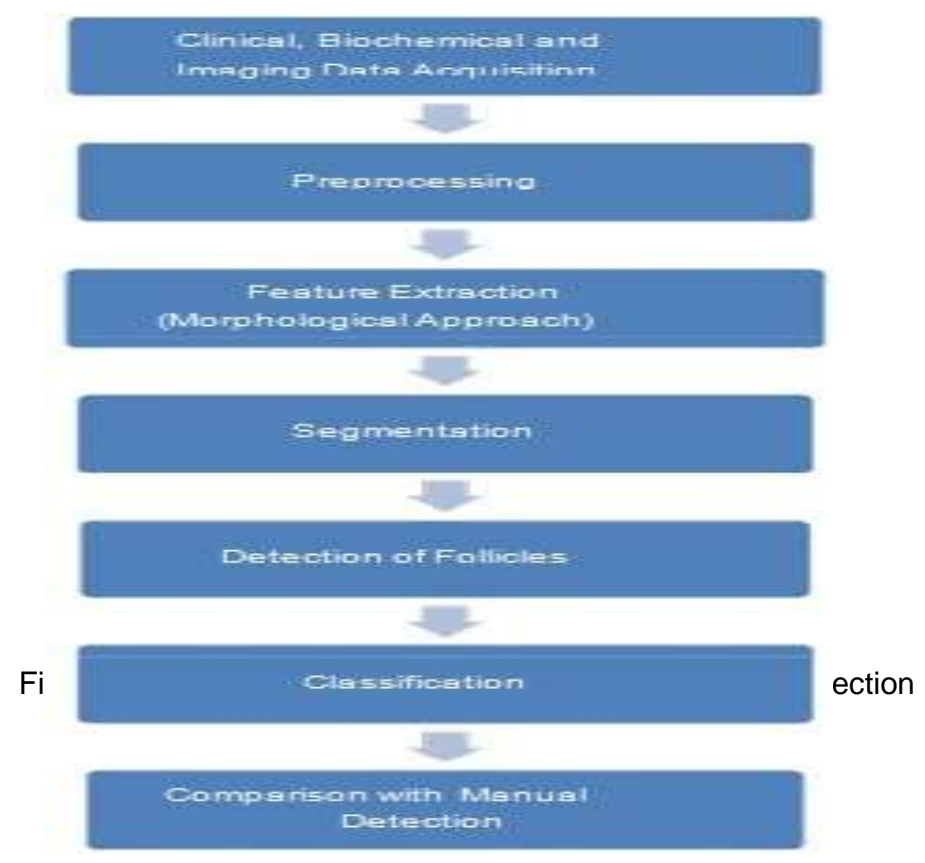

Fig 2 : Flow Diagram of Proposed Methodology 


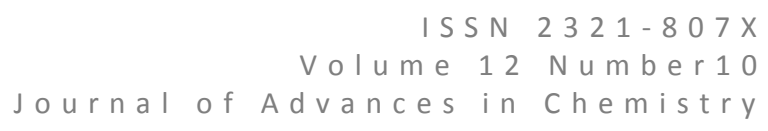

In this paper, automated detection of PCOS is addressed by combining all the three methods viz. clinical (BMI and cycle length), biochemical (FSH and $\mathrm{LH}$ levels) and imaging (calculating number of follicles present in the ovary). The transducer of procedure is $6 \mathrm{MHz}$ ultrasonic transducer fabricated by General Electric(GE).To enhance the nature of the images gathered, preprocessing procedures, for example, contrast enhancement and filtering are associated [1].Feature extraction is finished by multiscale morphological methodology utilizing Top-hat transform.Arrangement is completed utilizing Support Vector Machine Algorithm (SVM).

\section{Feature Extraction and Segmentation}

To diminish false identification, feature extraction is completed by utilizing Multiscale morphological methodology [6]. Here we are using Top-hat transform. Dark and white components can be extricated from the image and after that following image is subtracted from unique image to get contrast enhanced image.

In this algorithm the Top-hat transformation helps in the withdrawal of bright (or dark) features from the background by using morphological opening and closing process of a structuring element respectively. Image segmentation is used to identify desired features so that the resultant image is easier to evaluate [4]. For segmentation of ovarian ultrasound image, different edge detection techniques like Sobel, Prewitt and Canny edge detection can be utilized. In edge detection technique, points in image are recognized somewhere image brightness changes sharply or has discontinuities, as in image we get after binarization.
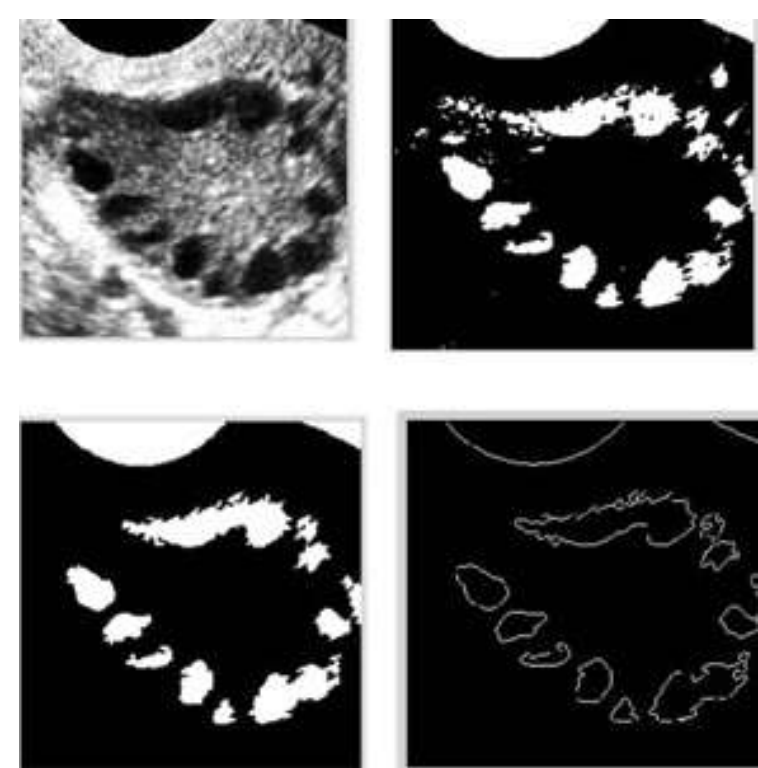

\section{Extracted follicles}
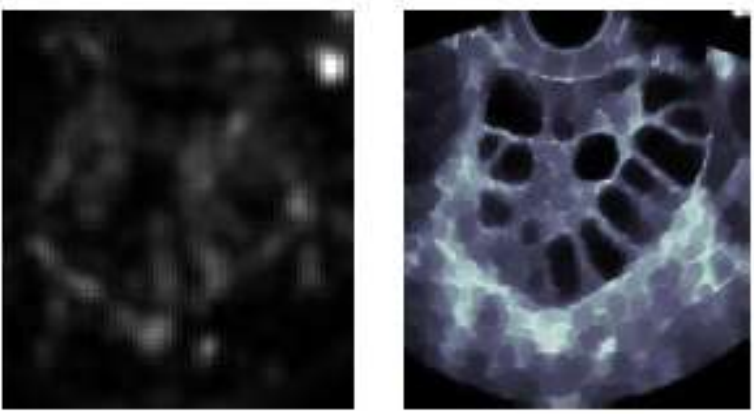

Saliency map

Fig 3 (a) Image obtained after feature extraction using bright top-hat transform (b) Image obtained after binarization (c) Image obtained for important follicle detection (c) Follicular boundalies detected after canny edge detection

\section{Detection of Important Follicles}

Large numbers of elements are recognized after binarization and edge detection. To isolate out needed follicles from identified features, criteria, for example, most extreme and least size of follicles, area of follicles, eccentricity and compactness must be added to the algorithm [9]. According to studied literature, follicular size reaches from $2-9 \mathrm{~mm}$ in width in PCOS influenced ovary and around $20 \mathrm{~mm}$ in normal ovary.

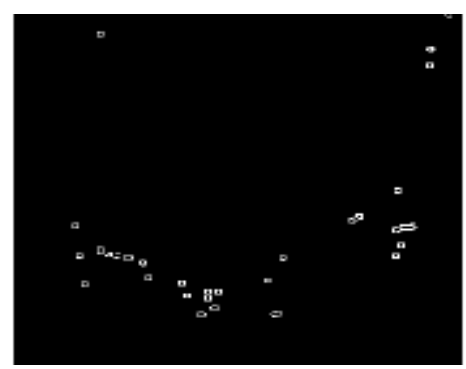

Fig 4 : Detected follicles 


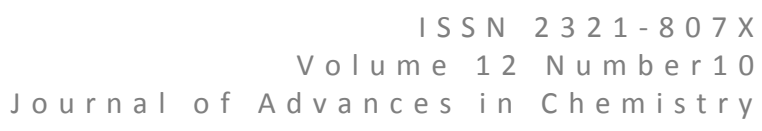

As a result, considering follicles are circular in shape, respective areas will be about 4-80 mm2 for PCOS ovary and about $314 \mathrm{~mm} 2$ for normal ovarian follicle. The value of eccentricity is equal to 1.

\subsection{OVARIAN TISSUES BY P63 TECHNIQUE}

Histopathology research center specialists use tissue tests for routine examination. These tissues are set up on biopsy slides utilizing shading chemicals. A study by [1] said that stain preparation (i.e. timing, concentration) and transmission of light assumes a key part for sectional thickness and in this manner; it is key to institutionalize the reagents and techniques during slide arrangement. Color uniformity is a significant factor for color image processing [8].

\section{A. Correcting Image objects}

A specific district may contain more than one protein and therefore; changing over images to grayscale won't give reasonable result because of the way that it might along these lines result the whole centralization of all proteins to a single region [2].Rather than utilizing a grayscale; shading image is a suitable decision for ovarian tissue investigation. One of the significant issues with digitized microscopic images is the non-uniform illumination which differs contrast. To attain uniform illumination all over the digitized color image is basically impossible even with the superlative bright-field microscopes which have the facility to reject most color balance problems. Hence, it is vital to correct image artifacts before image handling. There are numerous techniques to correct uneven-illumination issues for example correcting with blank image slides [2], using a filter operation [1], or using a mathematical a morphological operation [3].A filter operation or a mathematical morphological operation is measured to be the selected method.

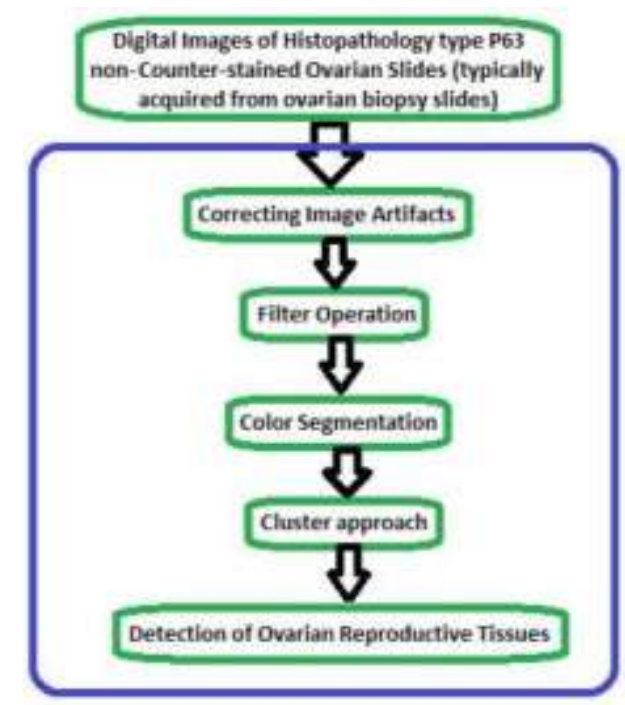

Fig 5: Block diagram for automated identification process

Test images utilized as a part of this study were firstly uncovered Gaussian low-pass channel to minimize artifacts issues as utilized as a part of prior studies [1] which recommended this as an appropriate alternative to minimize image artifact issues. So, proposed method for illumination correction is morphological operation. The HSV shading model was utilized [3] to right light minor departure from $\mathrm{V}$ channel. Last stage was the transformation back to RGB from HSV which gives the corrected image.

One of the significant issues with digitized tiny images is the non-uniform enlightenment which shifts contrast. Two of the critical variables in advanced shading imaging are shading parity and uniform light. An image having adjusted shading likewise has uniform brightening for all hues around the image. To accomplish uniform brightening everywhere throughout the digitized shading image is for all intents and purposes inconceivable even with the best brilliant field magnifying lens which have the office to dispense with most shading parity issues. In this manner, it is critical to right image antiquities before image preparing.

\section{B. Filter procedure}

Noise reduction is an essential pre-preparing venture before image segmentation. There are a few sorts of channels are accessible to evacuate noise. Be that as it may; for average image handling particularly for digitized histopathology ovarian regenerative tissue distinguishing proof mean and middle channels are prevalent [4]. Different studies [5] which have researched existing ovarian regenerative tissue distinguishing proof methodologies have connected one of these standard noise expulsion procedures. The middle channel is an outstanding methodology for image preparing to dispense with noises as it has better ability to save image edges than a mean channel. In any case, one of the significant disadvantages is, it brings about haziness impact. Determination of suitable cut-off sweep alongside a legitimate veil size would be helpful to minimize haziness. 


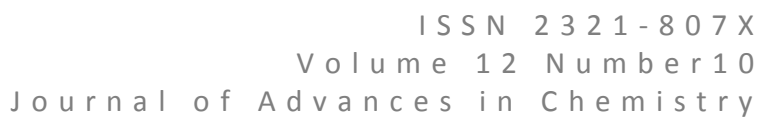

The ovarian tissue test images were presented to median filter to expel commotion. This strategy did not give tasteful result as the resultant image did not minimize fogginess totally as appeared in Fig.5.
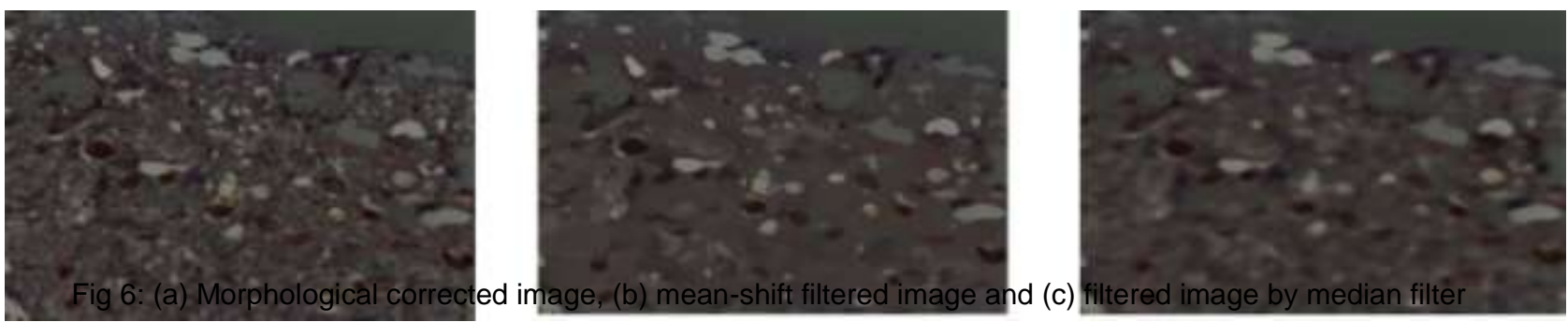

\section{C. shade Segmentation}

Pathology image segmentation particularly ovarian tissue segmentation is a troublesome assignment because of the way that they contain complex shape, size and shading. Existing exploration work demonstrates that diverse strategies including edge based technique [6], edge based strategy $[5,6]$ and by utilizing scientific morphology, for example, the watershed approach [6] can be material particularly to fragment grayscale images. For shading digitized images famous strategies are bunch based strategy (k-implies, mean-shift), Gaussian blend model based methodology, chart cuts, Markov irregular field based methodology [7]. To defeat the issues connected with tuning parameters mean-shift methodology is an appropriate decision as it is a non-parametric methodology which does not require any suppositions on the state of the conveyance anyway; it is computationally costly and tedious [7]. One study by [8] said that computational expense can be minimized for mean-shift approach notwithstanding utilizing a RGB shading images if quality is not the primary concern. Thusly, utilizing mean-shift approach both offices including brilliant result and decrease of computational expense is difficult to accomplish.

To minimize computational cost and to keep up top notch result one of the promising shading segmentation methodologies is measurable area combining [8]. This is computationally reasonable in-contrast with mean-shift approach anyway; it requires 2 parameters which incorporate $Q$ (free variable used to decide locale in pixels) and least district territory. Additionally, [8] said that for this methodology there are two particular disadvantages which incorporates standard deviation blunder and there is a possibility of square shaped shape areas after segmentation. Subsequently, to minimize the previously mentioned issues [8] has proposed to utilize least district region under or equivalent to 10.

\section{Cluster Approach}

There are various grouping methods accessible for the distinguishing proof procedure. Most methodologies require a precharacterized group parameter to perform bunching operation, for example, k-implies, DB-examine. As specified before, mean-movement is a non-parametric methodology [9] which does not require any pre-characterized bunch number. This was thought to be the best approach for the test images utilized as a part of this study. Nonetheless, a study by recommended that a co-fluctuation framework would be important to minimize MSE (mean square mistake) because of the way that all qualities for grouping are ordinarily in the middle of $0-1$ instead of unique worth. Decision of a cofluctuation grid is normally hard. Subsequently, this study changed the current system [5] keeping in mind the end goal to settle this issue. This study fused the middle co-ordinate values for every information point to wipe out the utilization of a co-difference framework. In addition, Gaussian piece and bit weight was joined to process the mean quality for every one of the information focuses that are accessible inside the bunch transfer speed. For this proposed changed grouping approach introductory bunch has been chosen as 0 as it is not known what number of groups can be found.
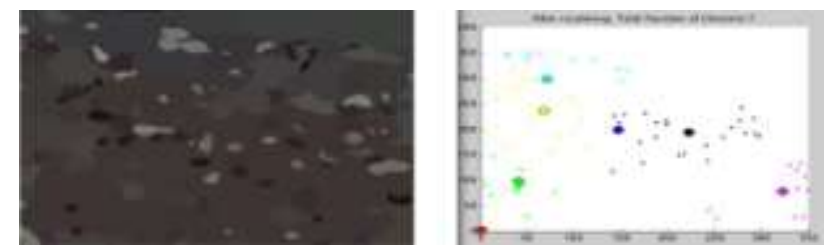

Fig 7 (a) Segmented image using modified region fusion approach and (b) total number of clusters (7 for this image) after using modified mean-shift clustering approach.

\section{CONCLUSION}

In this paper, we have tried to develop an automated method for Polycystic Ovarian Syndrome (PCOS) detection using follicle recognition and classification using SVM and ovarian reproductive tissues using type P63 (non-counter-stained) digitized images has been proposed. Image preprocessing (contrast enhancement and filtering) is used for improving quality of the image. Features are extracted using Multiscale morphological approach and bright top-hat transform. The image is binarized and segmented using canny edge detection technique. Important follicles are separated from other regions using area and eccentricity threshold. This study is novel as it is the first published study where type P63 noncounter stained human ovarian reproductive tissues were used. While other studies such as worked with human tissues using type PCNA but was limited in scope as used only small number of images. This study was extensive in using a 
large batch of test images. Furthermore, this approach is fully automated in comparison to other existing work with no human intervention required to assess new set of images and for different magnifications.

\section{REFERENCES}

1. H. Lyon, A. De Leenheer, R. Horobin, W. Lambert, E. Schulte, B. Van Liedekerke, et al., "Standardization of reagents and methods used in cytological and histological practice with emphasis on dyes, stains and chromogenic.

2. D. Magee, D. Treanor, D. Crellin, M. Shires, K. Smith, K. Mohee, et al., "Colour normalisation in digital histopathology images," 2009

3. S.Vijayprasath, R.Sukanesh, S.Palanivel Rajan, "Certain Investigations on Eye State Analysis for the Development of a Non-Intrusive Fatigue Detection System", European Journal of Scientific Research, ISSN No.: 1450-216X/1450202X, Vol. 93, No 3, pp.342-350, 2012.

4. K. Bapure, "Automated Image Analysis for Nuclear Morphometry Using H\&E and Feulgen Stains in Prostate Biopsies," 2012.

5. C. G. Loukas, G. D. Wilson, B. Vojnovic, and A. Linney "An image analysis based approach for automated counting of cancer cell nuclei in tissue sections," Cytometry part A, vol. 55, pp. 30-42, 2003

6. G. Landini and I. Othman, "Estimation of tissue layer level by sequential morphological reconstruction," Journal of microscopy, vol. 209, pp. 118-125, 2003.

7. S.Palanivel Rajan, R.Sukanesh, "Experimental Studies on Intelligent, Wearable and Automated Wireless Mobile TeleAlert System for Continuous Cardiac Surveillance", Journal of Applied Research and Technology, ISSN No.: 16656423, Vol. No. 11, Issue No.: 1, pp.133 - 143, 2013.

8. G. Wu, X. Zhao, S. Luo, and H. Shi, "Histological image segmentation using fast mean shift clustering method," Biomedical engineering online, vol. 14, pp. 24, 2015.

9. B. Fulkerson and S. Soatto, "Really quick shift: Image segmentation on a GPU," in Trends and Topics in Computer Vision, ed: Springer, 2012, pp. 350-358.

10. Y. Cheng, "Mean shift, mode seeking, and clustering," Pattern Analysis and Machine Intelligence, IEEE Transactions on, vol. 17, pp. 790-799, 1995.

11. S.Palanivel Rajan, R.Sukanesh, S.Vijayprasath, "Analysis and Effective Implementation of Mobile Based Tele-Alert System for Enhancing Remote Health-Care Scenario", HealthMED Journal, ISSN No. : 1840-2291, Vol. No. 6, Issue No. 7, pp. 2370-2377, 2012.

12. Affiliates of Medifocus.com. Medifocus guidebook: polycystic ovary syndrome. Silver Spring: Medifocus.com, Inc.; 2007.

13. S.Palanivel Rajan, R.Sukanesh, S.Vijayprasath, "Design and Development of Mobile Based Smart Tele-Health Care System for Remote Patients", European Journal of Scientific Research, ISSN No.: 1450-216X/1450-202X, Vol. No. 70, Issue 1, pp. 148-158, 2012.

14. Loizou CP, Pattichis CS, Christodoulou Cl, Istepanian RSH, Pantziaris M, Nicolaides "A Comparative evaluation of despeckle filtering in ultrasound imaging of the carotid artery" IEEE Transactions on Ultrasonics, Ferroelectrics and Frequency Control 2005;52:1653-69.

15. K Sheikdavood, P Surendar, A Manikandan, "Certain Investigation on Latent Fingerprint Improvement through MultiScale Patch Based Sparse Representation"Indian Journal of Engineering 13 (31), 59-64.

16. Sharvari S. Deshpande, Asmita Wakankar, "Automated Detection of Polycystic Ovarian Syndrome Using Follicle Recognition" 2014 IEEE International Conference on ICACCCT, ISBN No. 978-1-4799-3914-5/14.

P.S.Hiremath and lyothi R Tegnoor, "Automatic detection of follicles in ultrasound images of ovaries," proceedings of International Conference on Systemic, Cybernetics and Infonnatics- ICSCI09 (India, Hyderabad) 327-330.

17. B. Potocnik D. Zazula, "Automated analysis of sequence of ovarian ultrasound images, part I: segmentation of single 2d images", Image vision and Computing, vol. 20, no 3, 2002, pp 217-225.

18. S.Palanivel Rajan, K.Sheik Davood, "Performance Evaluation on Automatic Follicles Detection in the Ovary", International Journal of Applied Engineering Research, ISSN No.: 0973-4562, Vol. 10, Issue 55, pp. 1-5, 2015. 